\title{
A numerical model for the analysis of the locomotion of a cownose ray
}

\author{
Giovanni Bianchi* \\ PhD Student \\ Dipartimento di Meccanica \\ Politecnico di Milano \\ Milano, Italy 20156 \\ Email: giovanni.bianchi@polimi.it \\ Simone Cinquemani \\ Professor \\ Dipartimento di Meccanica \\ Politecnico di Milano \\ Milano, Italy 20156 \\ Email: simone.cinquemani@polimi.it \\ Paolo Schito \\ Professor \\ Dipartimento di Meccanica \\ Politecnico di Milano \\ Milano, Italy 20156 \\ Email: paolo.schito@polimi.it \\ Ferruccio Resta \\ Professor \\ Dipartimento di Meccanica \\ Politecnico di Milano \\ Milano, Italy 20156 \\ Email: ferruccio.resta@polimi.it
}

\begin{abstract}
Among all aquatic species, mantas and rays swim by flapping their pectoral fins; this motion is similar to other fishes in terms of efficiency, but it gives better maneuverability and agility in turning. The fin motion is featured by a traveling wave going opposite to the forward motion, producing a force thanks to momentum conservation. This article aims at understanding the swimming dynamics of rays, focusing on energy efficiency. A CFD model of the swimming motion of a cownose ray has been implemented in OpenFOAM, simulating the acceleration of the fish from still to the steady-state velocity using an overset mesh. In this analysis, the 1-DOF dynamics of forward swimming is solved together with the fluid velocity and pressure. The effect of frequency and wavelength of fin motion on thrust, power, and velocity has been investigated and an analysis of the vortices in the wake showed has been performed. The energy efficiency of a self-propelled body has been defined in a novel way and it has been calculated for different motion conditions. The results showed that batoid fishes swim with high energy efficiency and that they are a promising source of inspiration for biomimetic autonomous underwater vehicles.
\end{abstract}

*Address all correspondence related to ASME style format and figures to this author.

FE-21-1264, Bianchi, 1 


\section{Introduction}

Swimming is the first kind of locomotion that appeared on Earth. Aquatic organisms have been evolving through natural selection for millions of years, suggesting that their motion strategies have reached a great level of optimality [1]. This explains the interest of researchers and engineers in exploiting such mechanisms for the propulsion of autonomous underwater vehicles (AUVs), which could be employed for marine life observation, search and rescue operations, or environmental monitoring of aquatic environments. For the development of this technology, it is fundamental to understand the mechanisms underlying thrust production and the reasons why fish movements guarantee great maneuverability and low power consumption. Batoid fishes, an order including mantas and stingrays, swim by moving their large pectoral fins, this gives them a remarkable advantage in term of maneuvrability and stealth; furthermore, the endurance of batoid swimming suggests that their swimming motion is characterized by a high efficiency [2]. These characteristics are extremely desirable for AUVs, as they are typically designed to move with agility in narrow spaces and to roam underwater for a long time. The purpose of this work is to investigate the dynamics of batoid fishes swimming, that can serve as a starting point for bioinspired AUVs design. Batoid fishes locomotion belongs to the category of median paired fin (MPF), as they use their pectoral fins to propel themselves [3]. Fin motion basically consists in the formation of a chord-wise wave, which travels in a direction opposite to the fish motion. This wave pushes backward the surrounding fluid and the fish gains a forward thrust as a consequence of momentum conservation. This principle underlies the locomotion strategies of the majority of marine animals, whatever type of fin is responsible for thrust generation, and it has been deeply analyzed by Lighthill [4], Sparenberg [5] and Wu [6]. They provided an accurate explanation of the mathematics clarifying the principles of fish locomotion; however, their analytical approach is based on several simplifying assumptions that do not capture the complexity of the fin motion. Conversely, a numerical approach is more suitable to investigate the forces acting on the fins and the wake structure, relating them to the kinematic parameters of fin deformation. Computational Fluid Dynamics (CFD) is a very common tool used to investigate fish locomotion and, in particular, the swimming of batoid fishes. Previous studies include the work of Fish [7], who investigated the efficiency of steady-state swimming of myliobatoid fishes with an immersed boundary element method; the research of Liu [8], who analyzed the wake structure of a robot inspired to a batoid fish swimming at a constant velocity, the research of Zhan [9] about the gliding of a manta under the sea surface, and the numerical investigation of Sharp [10], who studied the forces acting on a fin performing an undulatory motion using Multi-particle Collision Dynamics. The dynamics of the interaction with water of a deformable fin has been explored by Liu [11] who modeled the fin as a series of panels connected by springs and by Wei, who performed a dynamic analysis of the motion of a Raja Eglanteria coupling the CFD with a dynamic solver [12]. Bottom et al. performed numerical simulations of swimming in stingrays analyzing vortices in the wake [13]. Similarly, Thekkethil et al. studied the hydrodynamics of batoid fishes describing the three-dimensional vortex structure, and they highlighted that the cownose ray has one of the highest thrust coefficients among batoid fishes [14]. Huang et al. performed a hydrodynamic analysis coupled with a complete 6DOF simulation of a bioinspired robot with undulating fins and two propellers, they have found a relation between fin flapping frequency and speed of the robot and they studied the stability of the robot during forward swimming and during manoeuvers [15]. The work presented in this article improves the results of the aforementioned researches analyzing the forward swimming of a cownose ray (Rhinoptera Bonasus) accelerating from rest to the steady-state velocity. The deformation of the fin is imposed, whereas the forward swimming motion is calculated together with pressure and velocity fields of the surrounding fluid. The wake structure and the results, in term of thrust, efficiency, and steady-state velocity, are then compared and put in relation to frequency and wavelength of fin deformation. The objective of this analysis is to explain the complex phenomena, which allow batoid fishes to have a high efficiency and to relate them to wavelength and frequency of fin motion. For this purpose, a new measure of efficiency has been defined, different from those found in literature, which allows to quantify the batoid fishes' performances. Some aspects of batoid fishes locomotion have been investigated by the aforementioned studies, the main contribution of this article is to relate the swimming performances, such as steady-state velocity, energy efficiency and thrust, to the kinematic parameters of fin locomotion. The paper is organized as follows: Section 2 provides a description of cownose ray geometry and fin kinematics; Section 3 outlines the CFD model used for these dynamic analyses; in Section 4 the structure of the wake is analyzed; in Section 5 a definition of the efficiency for a swimming fish is proposed; in Section 6 the swimming performances with different kinematic parameters are compared; finally, Section 7 is dedicated to conclusions.

\section{Fin geometry and kinematics}

Batoid fishes adopt a median paired fin (MPF) locomotion, generating thrust by moving their large pectoral fins [16]. Within the order of batoids, both fin oscillation and fin undulation have been recognized; nevertheless, there is not a neat distinction between the two swimming modes. In both categories, the fin motion can be regarded as a traveling wave. Undulation implies more than 0.5 waves along the fish body, whereas, if the number of waves is less than 0.5 , the motion of the fin falls into the oscillatory category [17]. Oscillatory locomotion gives advantages in cruising at high speeds in pelagic environments, whereas undulator fishes have better turning capabilities, and they are favored in maneuvering in narrow environments [3]. The number of waves characterizing batoid species covers all the continuum range from fully undulatory (Taeniura Lymma) to fully oscillatory (Manta Birostris). Moreover, many species can adjust their wave number according 
to the environment and the situation. Due to these differences, undulatory fishes have a disk-shaped fin, whereas oscillatory fishes have a more triangular fin. The cownose ray, the object of this study, is featured by an average wave number of 0.4 , being classified as oscillatory [17], and its motion is featured by a good trade-off between cruising speed and turning radius. This makes these fishes interesting for AUV design and development. Shape and dimensions of the fin of a cownose ray
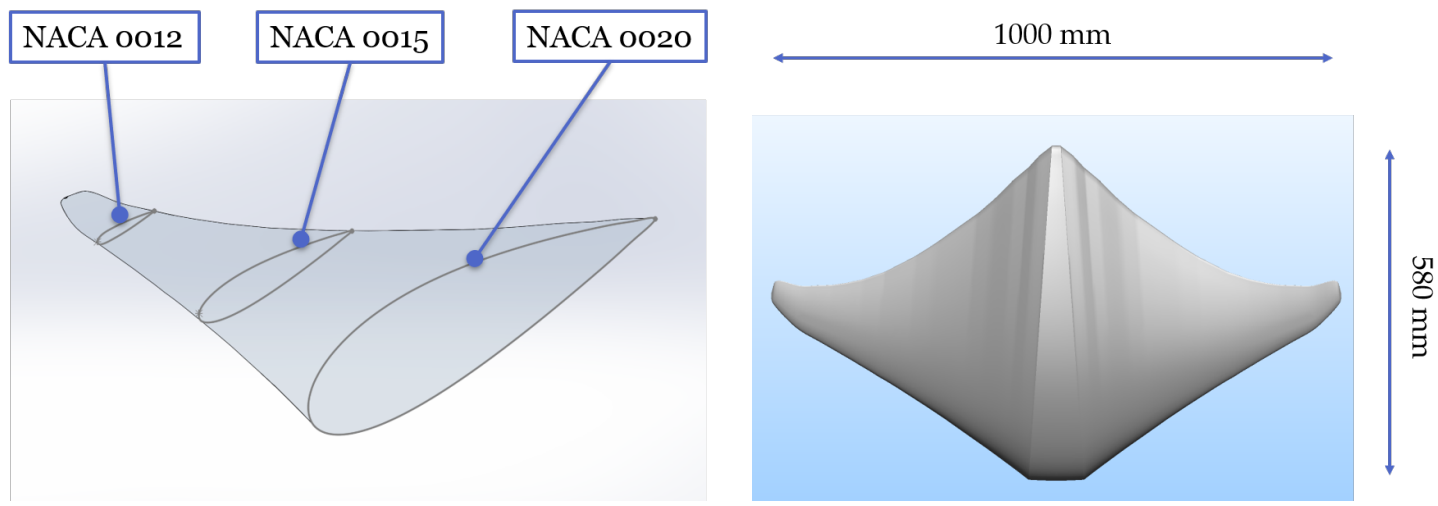

Fig. 1: CAD model of the cownose ray with its dimensions and the wing profiles that best approximate its shape

are taken from the work of Cai [18] [19] and they are shown in Figure 1. The cross-sections of the fin are approximated as a series of symmetric aerofoils smoothly tapering towards the fin tip: at the body center, the cross-section is approximated with a NACA 0020, at the fin mid-span with a NACA 0015, and near the fin tip with a NACA 0012. The mass of the fish is $4 \mathrm{~kg}$ [20]. The kinematics of batoid fishes locomotion has been described in detail by Russo [21] who developed a bio-mechanical kinematic model which allows to reconstruct the deformation of the fin at every time instant. Furthermore, they analyzed the skeletal structure of the cownose ray to quantify the parameters characterizing their fin motion. The cartilaginous structure of the fin is composed of several small radial segments connected with rotational joints. Considering the angle $\theta$ as the angle formed by a segment of the fin with the horizontal plane, $\theta$ of each segment is determined as follows:

$$
\theta(s, t)=\theta_{\max } s \sin (\phi x+\psi s-\omega t)+\delta s
$$

where $\theta_{\max }$ is the angle at the fin tip, $s$ is the position of the cartilage segment from the fin root, $x$ is the position from the leading edge, as shown in Figure 2, $\phi$ is the chord-wise wave number divided the body length of the fish, $\psi$ is the span-wise wave number divided the span length of the fin, $\omega$ is the frequency of fin flapping and $\delta$ is the mean value of the angle $\theta$ during a flapping cycle [21]. The wave numbers $\phi$ and $\psi$ are defined as follows:

$$
\phi=\frac{2 \pi}{\lambda_{x}} \quad \psi=\frac{2 \pi}{\lambda_{s}}
$$

where $\lambda_{x}$ is the wavelength of the wave propagating backwards in a longitudinal direction, whereas $\lambda_{s}$ is the wavelength of the wave propagating from the fin root to the fin tip [21]. Considering that the fin is composed of hundreds of segments and joints [21], it is possible to consider the fin motion as a continuous deformation of the fin, without losing accuracy in the representation of its geometry. Considering Eq. 1, s is considered as the curvilinear abscissa of each section of the fin, and $x$ is considered as the chord-wise coordinate. Equation 1 can be used to describe all the possible motions of a cownose ray fin. Nevertheless, the aim of this work is only to analyze the mechanism used to generate thrust in forward swimming, thus some simplifications can be made. These fishes usually reduce the downward stroke when they are hunting near the seabed. Nevertheless, in forward swimming, which is the most common motion, the upstroke and downstroke are of equal amplitude and fin motion is symmetrical. Hence, the coefficient $\delta$ can be neglected for this analysis. Moreover, it has been observed that the tip deflection lag, determined by the span-wise traveling wave, gives a minor contribution in thrust generation with respect to the chord-wise wave [22], therefore $\psi$ can be ignored. It has been hypothesized that the tip lag contribution consists in the reduction of losses due to the tip vortices [7]; however, this effect will be analyzed in a future work. Lying on these assumptions, Eqn. 1 becomes:

$$
\theta(s, t)=\theta_{\max } s \sin (\phi x-\omega t)
$$




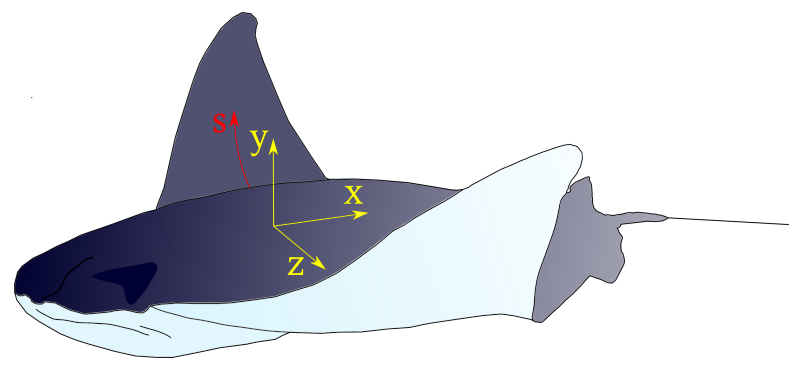

Fig. 2: Representation of the coordinates $x$ and $s$, used in Eq. 1, on the fin

and $y$ and $z$ coordinates of each point of the fin can be calculated as:

$$
y(s, t)=\int_{0}^{s} \sin [\theta(\sigma, t)] \quad d \sigma=\frac{1-\cos \left[\theta_{\max } s \sin (\phi x-\omega t)\right]}{\theta_{\max } \sin (\phi x-\omega t)}
$$

99

$$
z(s, t)=\int_{0}^{s} \cos [\theta(\sigma, t)] \quad d \sigma=\frac{\sin \left[\theta_{\max } s \sin (\phi x-\omega t)\right]}{\theta_{\max } \sin (\phi x-\omega t)}
$$

Eqn. 4 and Eqn. 5 represent a wave traveling in the opposite direction to the forward motion, pushing water backward.
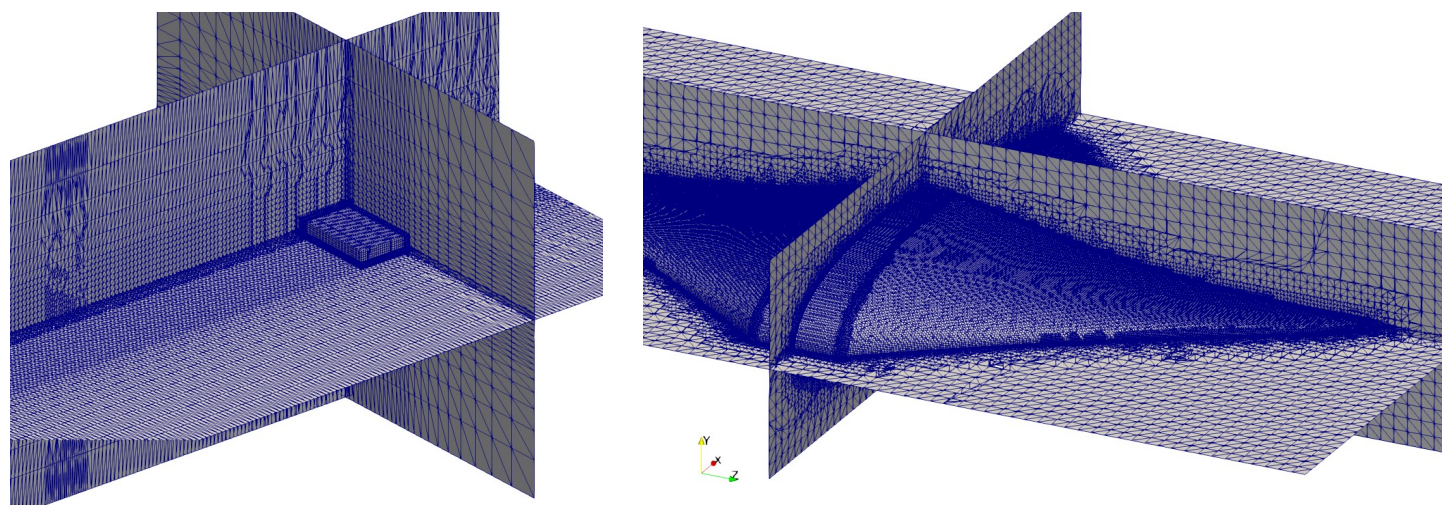

Fig. 3: Mesh used in the CFD simulations

Hence, for momentum conservation, the fish receives a forward thrust. The amplitude of this thrust depends on the speed at which water is accelerated by the traveling wave and a propulsive force can be generated only if the chord-wise wave travels faster than the swimming velocity of the fish [6].

\section{CFD model description}

A CFD model of a forward swimming cownose ray has been implemented in OpenFOAM. The 3D unsteady simulation is carried out using an overset mesh. It consists of two mesh grids merged together: the first encompasses all the domain and remains still, the second includes a portion of fluid around the fish and moves jointly with it. The two meshes occupy the same space and the software interpolates between overlapping elements to find a unique solution [23]. The motion of the internal mesh surrounding the fish is the result of the superimposition of two motions: forward swimming and fin deformation. As far as the fin deformation is concerned, every point of the mesh surrounding the fish is moved according to Eqn. 4 and Eqn. 5, resulting in a mesh deformation correspondent to the motion of the fin. This motion depends only on the kinematics of fins, which are imposed. On the other side, for forward swimming, the fish and its surrounding mesh are treated as a rigid body. The dynamics along the longitudinal axis of the fish are solved with a Newmark solver featured 
by $\beta=0.25, \gamma=0.5$ [24]. The only degree of freedom of the fish is the forward displacement, whereas all the others DOFs are constrained. Therefore, every time step the fins are moved, deforming the internal mesh, the solver calculates the forces acting on the fins and it moves forward the fish. The solved equations are URANS and the solver used in OpenFOAM is overPimpleDyMFoam, capable of solving unsteady, incompressible, turbulent flows with an overset mesh. The domain is shown in Figure 4, the fixed mesh is shown in blue and the moving mesh in red. The background domain is composed of

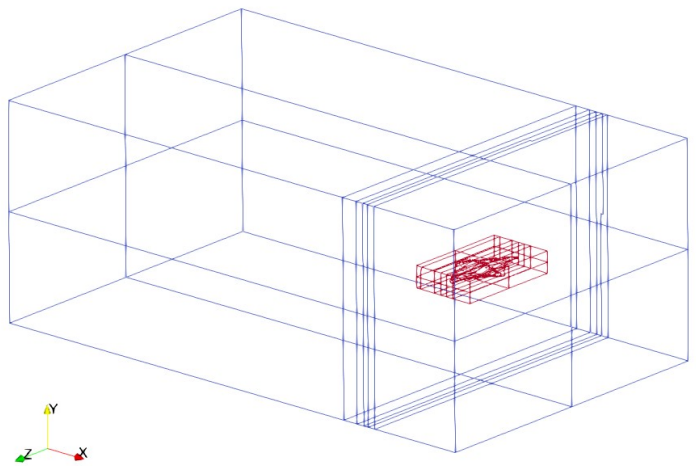

Fig. 4: Domain of the CFD simulations, differentiating the two meshes with two colors

$300000(200 \times 30 \times 50)$ hexahedral cells, with a gradual refinement towards the zone that is occupied by the moving mesh. Its dimensions are $5 \mathrm{~m} \times 3 \mathrm{~m} \times 2 \mathrm{~m}$ and it is shown in Figure 3a. The moving mesh is obtained using snappyHexMesh on a $0.6 \mathrm{~m} \times 0.2 \mathrm{~m} \times 1.1 \mathrm{~m}$ block, with 3 levels of refinement on the surface of the fish, resulting in a mesh of 1462199 cells, shown in Figure 3b. To reduce interpolation errors cells at the boundary of the moving mesh have similar dimensions to cells in the background. The results of the grid sensitivity analysis are shown in Figure 5a, where the velocities obtained with different mesh grids are shown. The coarsest mesh has two levels of refinement on the fish surface and the cells in the background are twice as large as the cells of the chosen mesh, whereas the finest mesh grid has four levels of refinement and its cells are twice as small. It is possible to note that the results obtained are independent of mesh size, since the average error between the normal and the finest mesh is $1.25 \%$. In Figure $5 \mathrm{~b}$ the surface of the fish is colored with $\mathrm{y}+$ for the normal

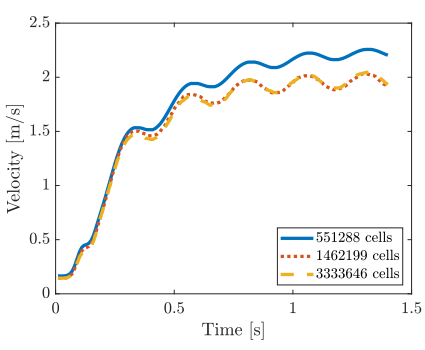

(a) Velocity of the fish obtained with mesh cells of different sizes

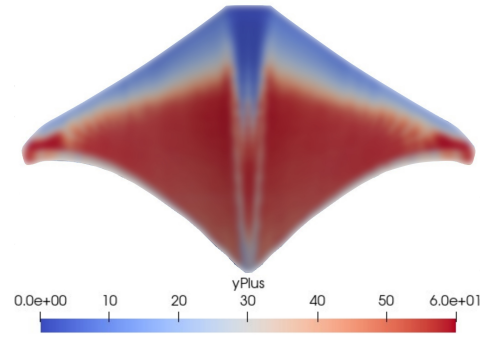

(b) $y+$ on the surface of the fish

Fig. 5: Mesh convergence and y+

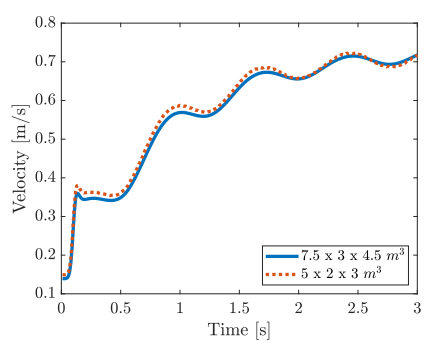

(c) Velocity of the fish obtained with domains of different sizes

mesh: the values are higher than 30 and standard wall functions are used with a $k-\omega S S T$ turbulence model. A similar analysis has been performed to assess the independence of the results from the domain size. The results are compared in Figure 5c, where it is possible to note that by increasing the domains size, the difference in velocity is negligible. For this analysis, the sizes of the overset domain have been changed with the same proportions as the background domain. The analysis of independence of the domain size is performed on a different simulation from the analysis of the mesh size. At the initial time step the fins are in their undeformed configuration and pressure is zero all over the domain. The velocity has been set to a very small fixed value of $1 \times 10^{-3} \mathrm{~m} \mathrm{~s}^{-1}$ on one extremity of the boundary; zero gradient velocity boundary conditions are applied to the other sides of the domain, and a moving wall velocity condition is applied on the surface of the cownose ray. Pressure is set to a fixed value of zero on one extremity of the domain and a zero gradient condition is applied 
downstroke in the plane at mid-span of the fin, shown in Figure 7a. The fin motion creates a large area of negative pressure

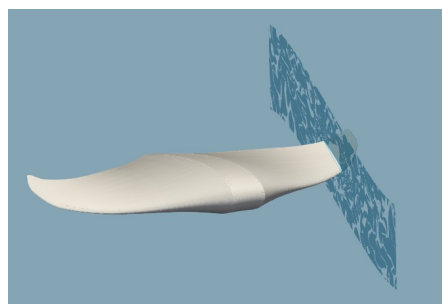

(a) Plane at mid span of the fin

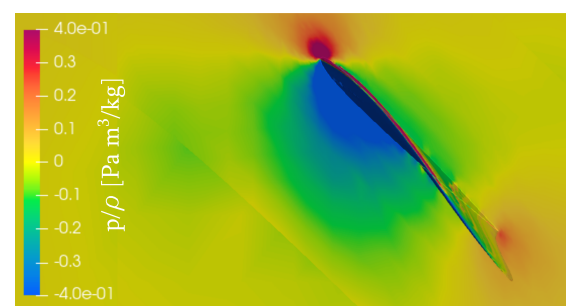

(b) Upstroke

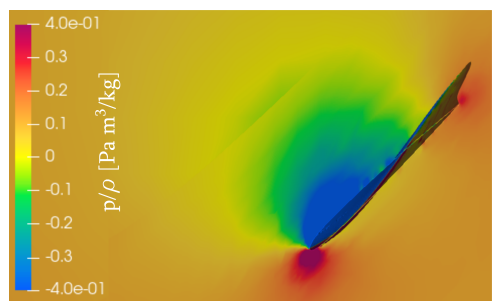

(c) Downstroke

where $\omega$ is the frequency of flapping, $A$ is the amplitude of fin-tip displacement and $L$ is the chord length of the fin. The Reynolds number results in $\sim 10^{5}$ for all the analyses.

\section{Structure of the wake}

The description of the thrust generation principle and of the structure of the wake is performed considering showing he simulation with $\omega=4 \pi \mathrm{rad} / \mathrm{s}$ and $\lambda_{x}=2.5 \mathrm{BL}$, but the qualitative description of the results can be extended to all the cases analyzed in this work. Thrust generation is concentrated on the external parts of the fins, where the motion is of higher ection divided the area of the element. A negative value, shown in blue, means a contribution to thrust, and a positive value, shown in red, represents a contribution to drag. Figure 7 illustrates the pressure distribution during upstroke and

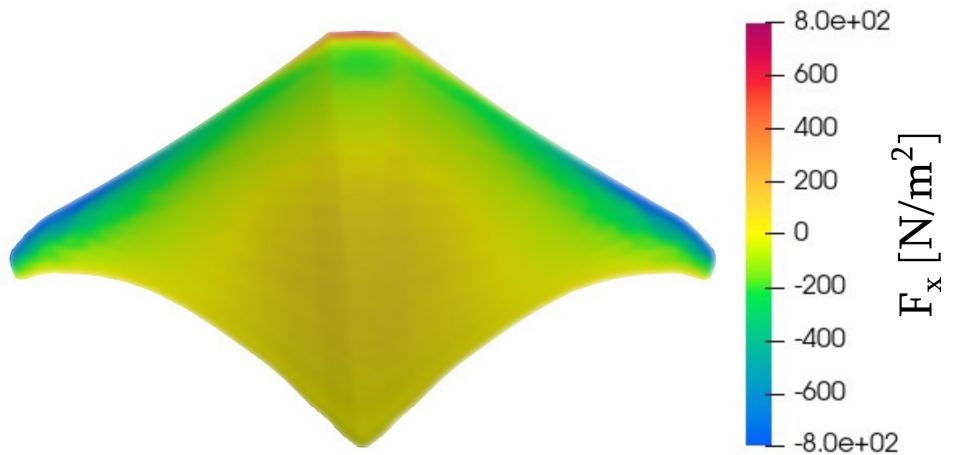

Fig. 7: Pressure distribution around the fin during upstroke and downstroke on a plane perpendicular to the fin span axis 
in front of the fish, which is pushed forward by this pressure difference. Observing the pressure distribution on the fins and the time profile of thrust, it is possible to notice that a propulsive force is generated during both upstroke and downstroke with equal values because both the profile and the fin motion are symmetric. Hence, during a flapping cycle thrust generation is subject to a sinusoidal variation with a double frequency with respect to the frequency of flapping. The generation of propulsive force is proportional to the fin velocity; thus, it is maximum in the mid-point of flapping and it is minimum at the upper and lower limits of its stroke.

A null thrust is generated when the fin tips reach the top and bottom extremities of their motion, whereas thrust is maximum in the middle of their motion. When the fin is at one end of its stroke, the velocity of its motion is zero, thus it cannot impress any momentum to the surrounding water, so it does not generate any thrust. This can be observed in Figure 8a, showing the variation of thrust over four periods of fin flapping. It is possible to note that at the beginning, when the fish is accelerating, there is a propulsive net force over one period, whereas, when it has reached the steady state, the average force over one period is zero. As shown in Figure 8b, steady-state velocity is reached after few cycles and it follows the same

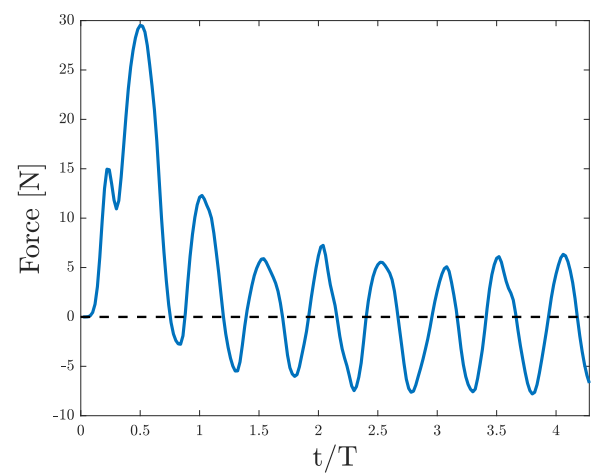

(a) Force in the sw0 imming direction during 4 periods

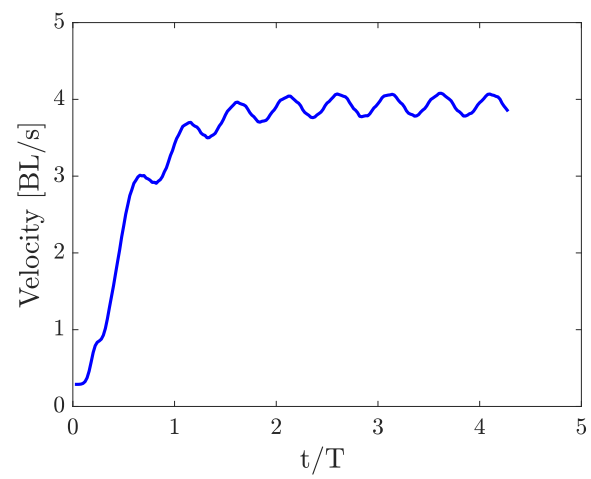

(b) Velocity of the fish

Fig. 8: Force in the swimming direction and velocity of the fish

sinusoidal variations as thrust. When the steady-state is reached the oscillations around the average velocity do not exceed $5 \%$ of the average velocity. The velocity of the fish is adimensionalized with respect to its dimensions and it is shown in body length per second (BL/s), where the body length is $0.58 \mathrm{~m}$, as shown in Figure 1 . To understand how the fluid is moved by the fins and why a thrust is generated, it is important to investigate the structure of vortices in the wake. The vortices in the wake are shown in Figure 9, seen from different perspectives. For each fin, it is possible to divide the wake into three different parts:

\section{- fin tip vortices}

\section{- vortices shed by fin motion}

\section{- center of the body}

The vortices in the wake are very similar to those obtained by Fish et al. [7]. The only difference is the absence in their work of the vortices near the center of the body. This could be due to the fact that they are simulating a different species of batoid fishes, which has a larger body than the cownose ray, thus the fluid motion around the body is less affected by fin flapping. An interesting result of this analysis is the comprehension of the mechanism allowing high efficiency of these fishes that is based on vortices shedding. Like many other fishes, cownose rays shed a vortex in the wake at each half flapping period, every time the fin reaches the top or the bottom of its motion. Such vortices rotate clockwise or counterclockwise depending on whether the fin has performed an upstroke or a downstroke [8]. Observing the wake structure in a plane perpendicular to the fin span shown in Figure 11, it can be noticed that vortices are arranged like in a reverse Karman Street, with counterclockwise vorticity on the top and clockwise vorticity at the bottom, as the fish swims leftwards. This arrangement of vortices produces a sort of propulsive jet in the center of the wake, whereas there is a counterflow in the surrounding volume. In the case the fish swims at a constant speed, jet stream and counterflow are balanced and the net stream-wise momentum in the wake is null $[4,26]$. Each fin section is moving like a heaving and pitching airfoil, which produces a reverse Karman street in the wake for a flow with a Reynolds number $\sim 10^{5}$, similar to the one of batoid fishes swimming. [27]. The vortex detachment mechanism is illustrated in Figure 10, where a section perpendicular to the span axis of the fin is shown at different time instants, with the fish swimming from the right to the left. The first time step is shown on the right: 

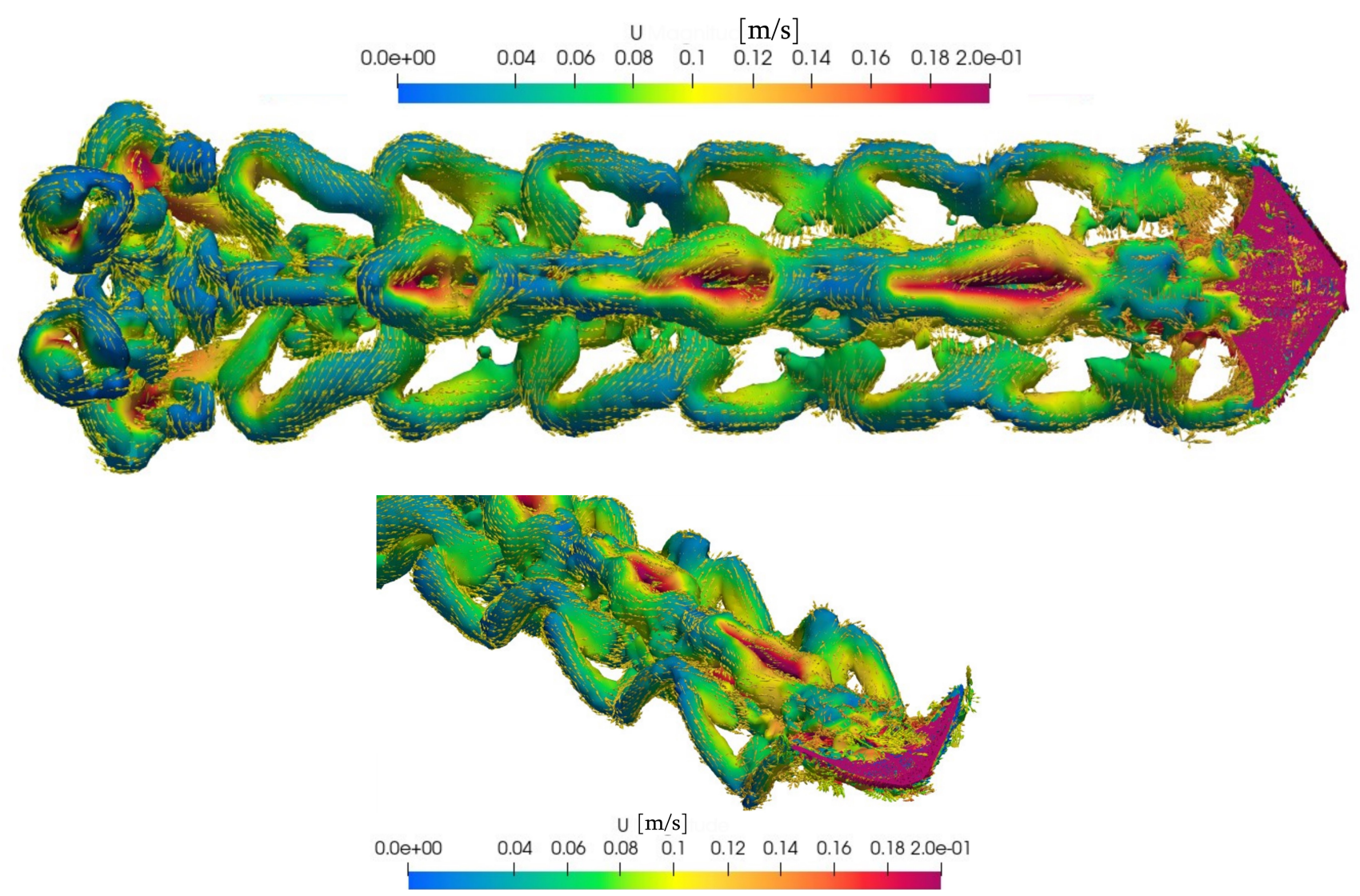

Fig. 9: Contour of surface $\lambda_{2}=0$, with vorticity vectors

(a) the fish is swimming leftwards, and it is performing an upstroke. On the lower side of the fin, a negative pressure area is formed, therefore water from the top side flows on the opposite side rotating around the leading edge of the fin. On the rest of the fin, the water is pushed upwards and backward following the motion of the traveling wave.

(b) When the fin reaches the upper extremity of its motion its velocity is null, as it is about to reverse the vertical direction of fin flapping. Hence, the difference of pressure between the two sides is absent and the vortex around the leading edge disappears, while water behind the trailing edge has an upward and backward velocity, impressed by the fin at the previous time instant.

(c) Then, when the fin begins its downward motion, the flow surrounding the fin is opposite to the one described in the first time instant, and the water behind the trailing edge is pushed downwards. Just behind, the water still conserves its upward velocity given by the upstroke, thus a vortex forms.

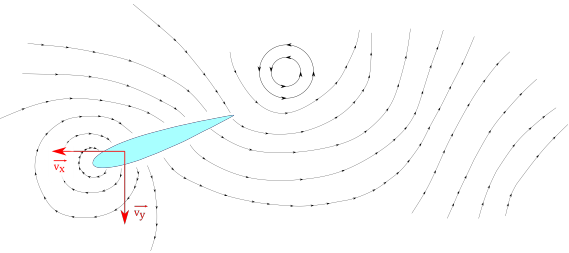

(c)

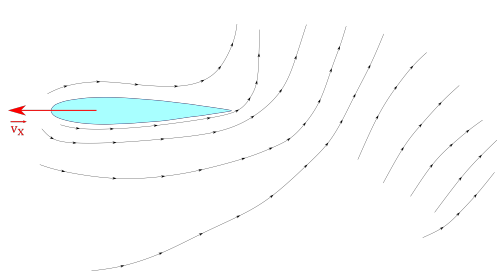

(b)

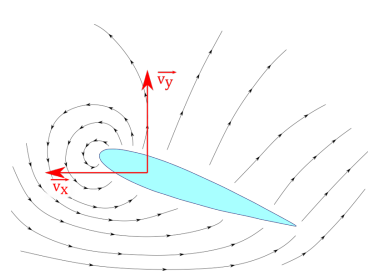

(a)

Fig. 10: Sequence of time steps during a quarter of flapping cycle, highlighting the detachment of one of the vortices forming the reverse Karman street

This mechanism of vortex formation is the same highlighted on a heaving and pitching airfoil $[28,29]$. Although vortices in the wake are a cause of dissipations, it is unavoidable that some vortices are generated when a body moves periodically 
in a viscous fluid. The vortices highlighted in the previous paragraph are a direct consequence of this kind of motion, responsible for thrust production [4,27]. Therefore, although it is desirable to keep them as small as possible, they are the result of thrust-producing motion of high energy efficiency. In Figure 12 it is also evident that during acceleration in the

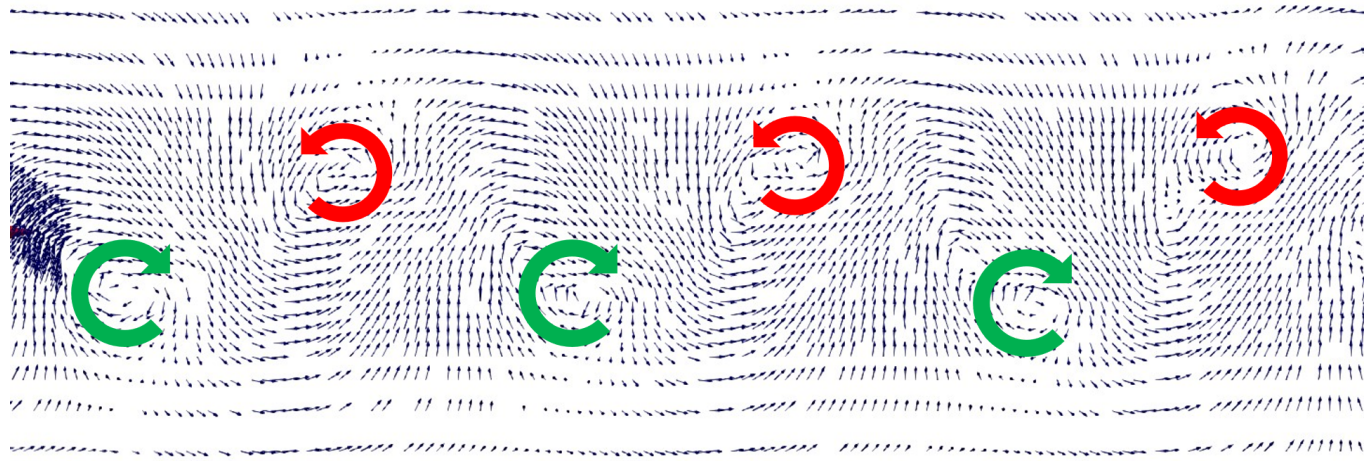

Fig. 11: Vortices in the wake

wake a momentum surplus in the opposite direction to swimming is present, remembering that the fish is swimming from the right to the left. On the top, velocity vectors in the wake are shown: water is pushed backwards by fin motion and that
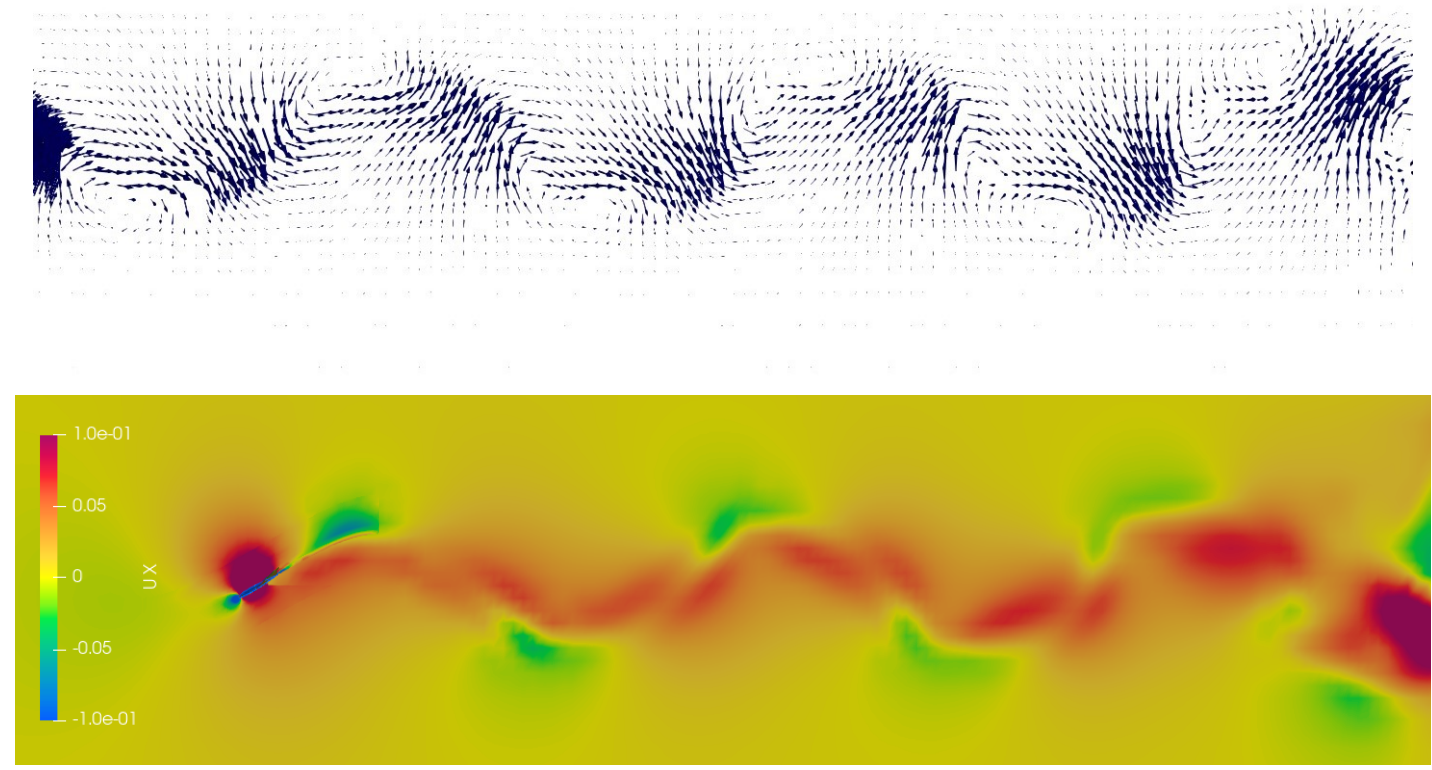

Fig. 12: On the top velocity vectors; at the bottom velocity component in the swimming direction

alternately upwards and downwards following the fin displacement. In the beginning, when the acceleration is greater, the amplitude of velocity vectors is higher. At the bottom, the velocity component in the swimming direction is presented in a color scale. It is possible to note that in the center a propulsive jet stream is present, whereas on the top and at the bottom, in correspondence of vortices, some water moves in the opposite direction. Another vortex is shown in Figure 13, where the velocity vectors are drawn on the plane shown in 13a. It is generated around each fin tip due to the difference of pressure between the two sides of the fin. During downstroke, the fin motion creates negative pressure on the upward-facing side, 
thus the fluid on the downward-facing side tends to rotate around the fin tip and move to the other side generating a vortex. The same mechanism is repeated during downstroke with a vortex rotating in the opposite direction. The generation of this vortex occurs only behind the fin tip because of the shape of the fish because the spanwise velocity of water that tends to move on the opposite side is smaller than the forward velocity of the fish divided the tangent of the sweep angle of the fin. The curvature of the fin is optimized to reduce the strength of this vortex. The high curvature of the fin makes its tip to act as a winglet and to obstruct the passage of water from one side to the other. Finally, another vortex is present in the center

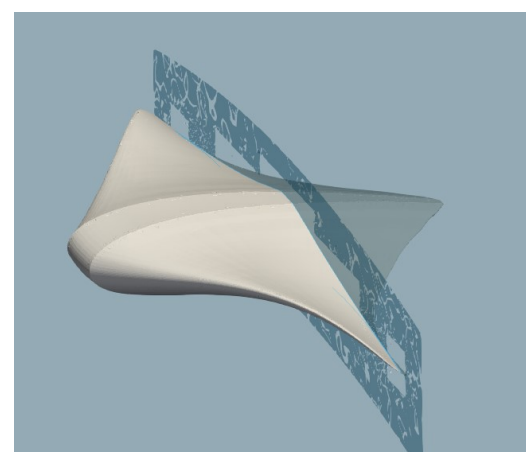

(a) Plane just behind the fin tip

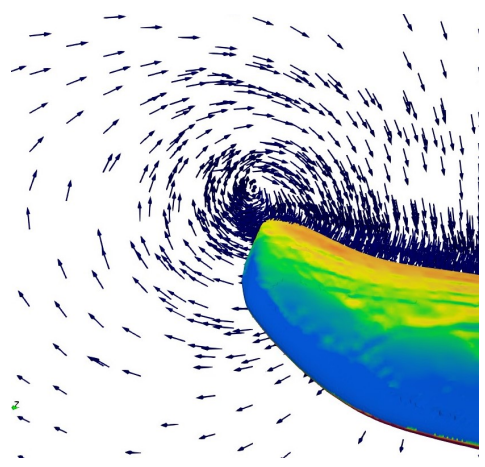

(b) Fin tip vortex

Fig. 13: Velocity vectors of the vortex around the fin tip during a downstroke

of the body, presented in Figure 14. Its presence is due to the alternate motion of the fin tips inwards and outwards during their motion. These vortices rotate in opposite directions on the two sides of the fish and they reverse their vorticity between upstroke and downstroke. The obtained results are consistent with the results obtained experimentally by Clark et al. [30]

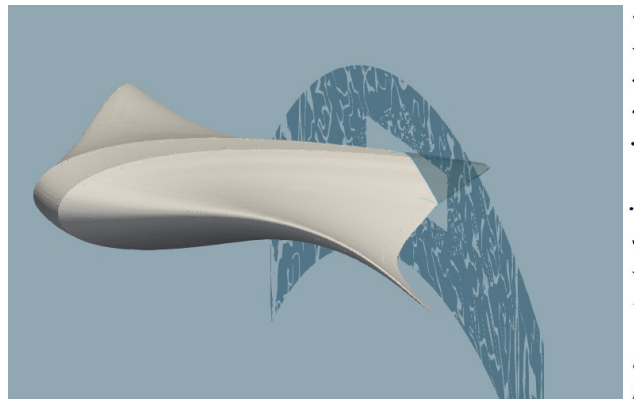

(a) Plane near the tail of the fish

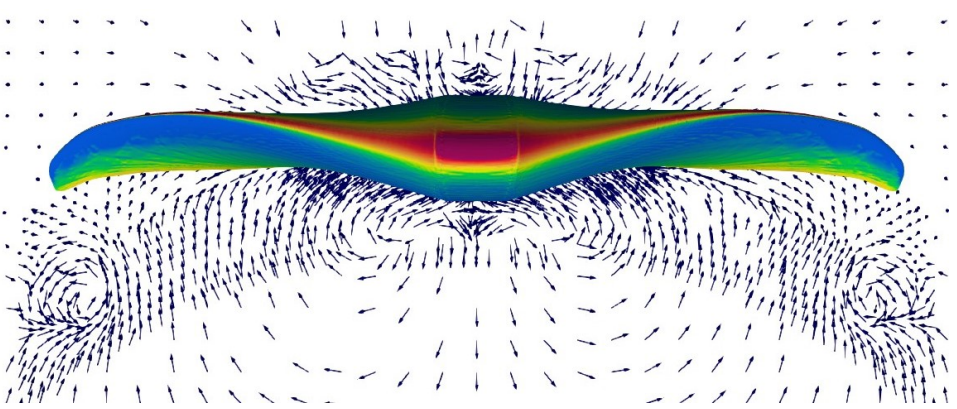

(b) Central vortex

Fig. 14: Velocity vectors of the vortex on the central part of the fish body

and by Dewey et al. [31], who performed experiments on a batoid-inspired fin actuated in a traveling wave motion. They have highlighted that vortices in the wake have an alternate sign and they are arranged similarly to a reverse Karman street and they have put in evidence a vortex around the fin tip. Conversely, the results differ significantly from those obtained numerically by Bottom et al. [13], since they have studied a stingray, whose fin has a different shape from the cownose ray and which undulate their fins with a much shorter wavelength.

\subsection{Leading edge vortex}

A vortex on the leading edge (LEV) of the fins is a typical feature of animals swimming with a lift-based propulsion, like carangiform and thunniform fishes $[13,32]$. In this kind of locomotion the fin acts as a heaving and pitching airfoil, with a high angle of attack, which generate a lift force in the front of the fin that pulls the fish forward. The presence of a stable leading edge vortex is very common in nature, since it enhances lift generation, and it is observed also on the wings of flying insects [13]. Cownose ray fin motion can always be described by Equations 4 and 5, but according to the wavelength the motion can be oscillatory or undulatory, and only oscillatory locomotion, in general, is characterized by a leading-edge 
vortex [32]. In this work the swimming performances with different wavelengths are compared, and the influence of this parameter on the LEV is discussed. In Figure 16a the streamlines of absolute velocity on the fin tip for a short wavelength are shown during a downstroke. It is possible to observe the vortex around the fin tip, with the fluid moving in a lateral direction, passing from the bottom side to the top side of the fin, but there is not any vortex on the leading edge, as the streamlines of the fluid passing around the leading edge are very large. Looking at Figure 15b, where the relative velocity vectors are

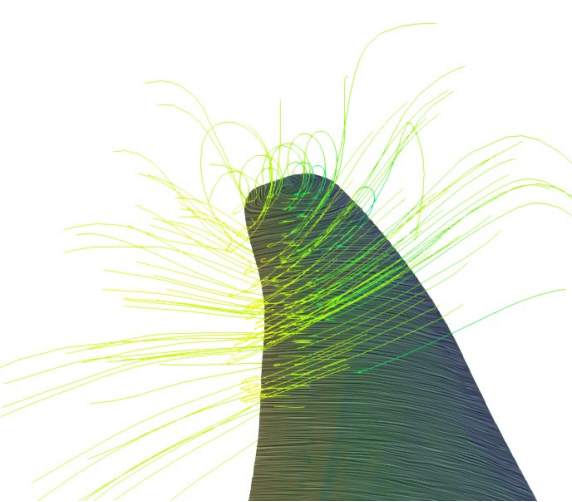

(a) Streamlines of absolute velocity around the fin tip for $\lambda=$ $1.67 B L$

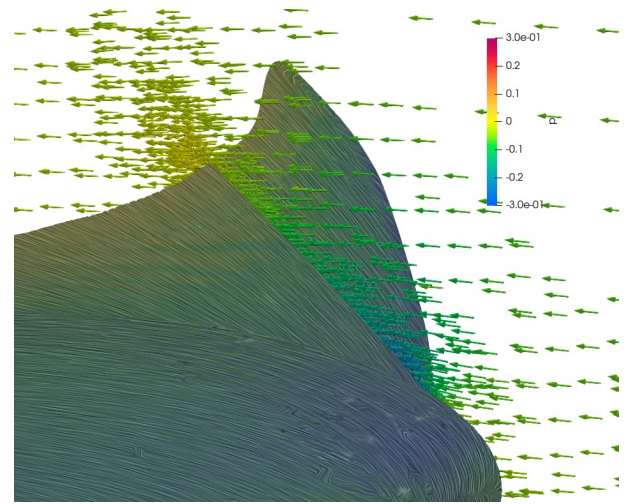

(b) Relative velocity vectors near the fin tip for $\lambda=$ $1.67 B L$

Fig. 15: Velocity vectors and streamlines near the fin tip for $\lambda=1.67 B L$

represented, the same observations can be done, since the velocity vectors always remain almost parallel to the swimming direction. The relative velocity is calculated as the difference of the velocity of the fluid and the forward swimming velocity of the fish, it is different from the relative velocity between each section of the fin and the fluid, since the fin is moving also in a vertical direction. Conversely, when the wavelength is larger and the motion is more oscillatory, a vortex near the leading edge is formed. The streamlines of absolute velocity around the fin tip during a downstroke are shown in Figure ??, it is possible to observe that around the leading edge a vortex is formed. The water near the leading edge flows from the top side to the bottom side and it rotates generating a vortex, connected with the vortex around the fin tip. This vortex can be

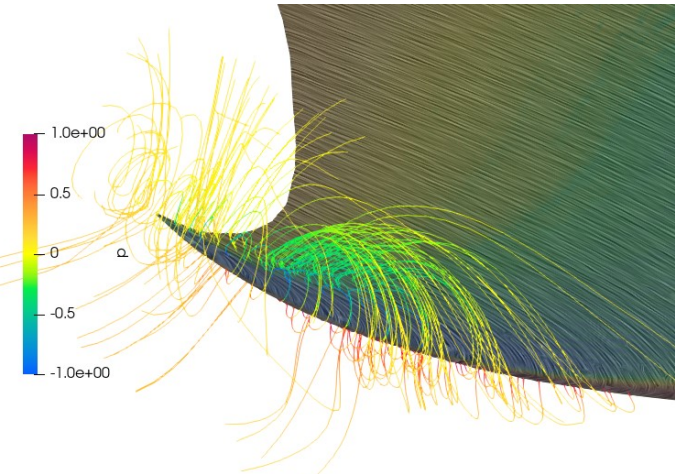

(a) Streamlines of absolute velocity around the fin tip for $\lambda=$ $20 B L$

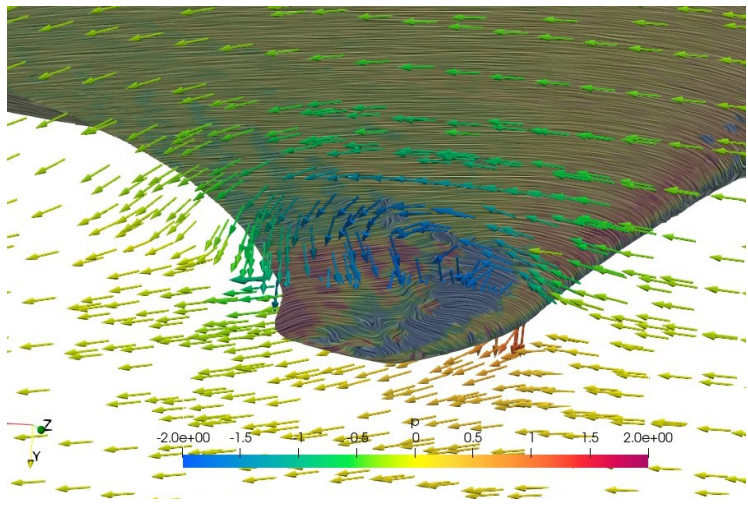

(b) Relative velocity vectors near the fin tip for $\lambda=20 B L$

Fig. 16: Velocity vectors and streamlines near the fin tip for $\lambda=20 B L$

observed also in Figure 16b, where the relative velocity vectors are displayed. This vortex is responsible for the generation of a low pressure area at its center, near the leading edge, which enhances thrust. The formation of a LEV for oscillatory fin motion of batoid fishes was also observed by [33], who studied the vortex formed by the fin motion of a manta, characterized by a longer wavelength than the cownose ray. They also have highlighted that the LEV strength increases with an increasing wavelength. 


\section{Efficiency of fin propulsion}

The traditional definition of propulsive efficiency is appropriate to systems where the propeller is separate from the main body, thus it is straightforward to distinguish the thrust component from drag. Conversely, in the case of fish swimming, fins deformation is responsible for thrust and drag at the same time, because the drag force depends also on the angle of attack of the fin, which is variable during fin motion. Thus, using the traditional Froude efficiency, shown in Eqn. 7, the resulting efficiency for steady-state swimming of a fish would be always zero because the net acting force in the swimming direction over a period is null [34], as thrust and drag both act on the same surface and at the steady-state they are equal and opposite. Since the swimming motion of a fish is unsteady, the efficiency can only be evaluated as the ratio between the average of output and input power over a period.

$$
\eta=\frac{\int_{T} F_{x} U_{x} d t}{\int_{T} P_{i n} d t}
$$

Hence, a different definition of efficiency is needed to quantify the energetic performances of a swimming fish. A parameter widely used in biology to measure the energy efficiency of different animals is the cost of transport (COT), which is defined as the energy spent per unit distance traveled [35]. Since this coefficient is dimensional and it depends on the mass of the animal, it is useful to compare different gaits of the same fish, but it does not provide information about the absolute efficiency of the motion.

To quantify the propulsive efficiency of a fin, it is needed to separate the contributions of thrust and drag, that act together on the same body. In this work, a different definition of efficiency is provided, which is suitable for self-propelled bodies like swimming fishes. Since the data coming from the simulation allow to distinguish the contribution of pressure and viscous forces, it is possible to give a proper definition of efficiency. The input power $P_{i n}$ is the power spent by the fish to move its fins, so it is obtained as the product between the forces acting on the fins and the relative velocity of the fins with respect to the fish body $\mathbf{v}_{\text {rel }}$, i.e. the vertical and lateral components of velocity (Eqn. 8). The forces acting on each infinitesimal element of the fin are the pressure force $p \mathbf{n}$, where $\mathbf{n}$ is the normal versor of the fin surface, and the viscous tangential stresses $\tau$.

$$
P_{\text {in }}=-\int_{A}(p \mathbf{n}+\tau) \cdot \mathbf{v}_{\text {rel }} d A
$$

The useful output power $P_{\text {out }}$ is the result of the pressure acting on the fins projected on the swimming direction, whereas the pressure acting on the rigid central body and the tangential stress acting on the whole surface contribute to the wasted power (9).

$$
P_{\text {out }}=\int_{A_{\text {fins }}} p v_{x} n_{x} \quad d A
$$

This definition of efficiency is indicative of how much power contributes to generating a force propelling the fish in the swimming direction. The tangential forces acting on the surface are the result of dissipative viscous effects and the pressure force acting on the central body is always resisting forward motion. In Figure 17 the contribution of each of these forces during few periods can be observed. When the fish has reached a steady-state velocity the total force in the swimming direction $(x)$ oscillates around a zero mean value. It can be decomposed in a positive contribution given by the pressure force acting on the fins and in a negative contribution given by the tangential forces and by the pressure acting on the central body. As it is possible to observe, when the fins reach the top or the bottom of their motion, they change direction and, in this instant, their velocity is zero. Therefore, they do not generate any useful force on the surrounding fluid, and only drag is present.

\section{Influence of kinematic parameters on swimming performances}

In this work, several dynamic analyses have been carried out changing frequency and wavelength of fin motion to investigate the performances of these fishes and understanding the "secret" of its efficiency. The amplitude of fin flapping is the same for all simulations.

\subsection{Effect of wavelength}

Figure 18a shows the velocity of the fish with different wavelengths. The cownose ray is featured by an oscillatory swimming and it moves its fins with a wave traveling at 2.5 body-lengths (BL) per second at about $1 \mathrm{~Hz}[17,21]$; however, 


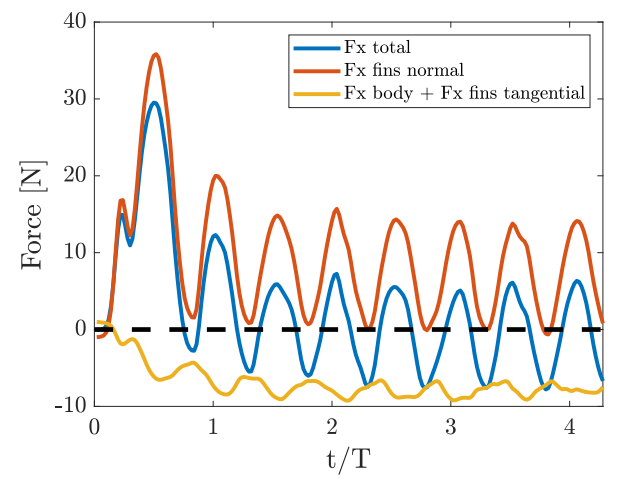

Fig. 17: Forces acting on the fish along the swimming direction

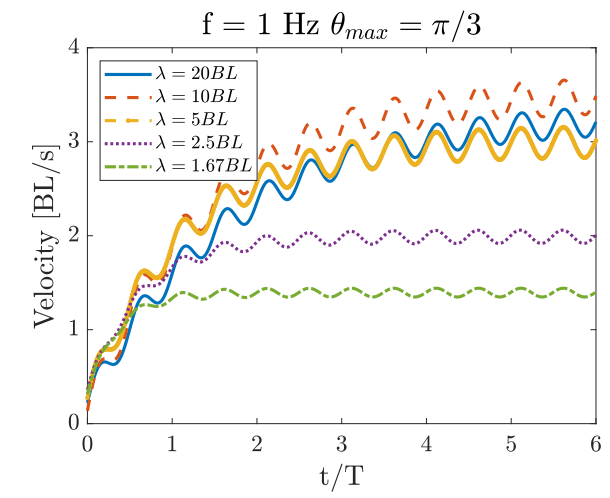

(a) Forward velocity of the swimming fish for different wavelengths at $1 \mathrm{~Hz}$

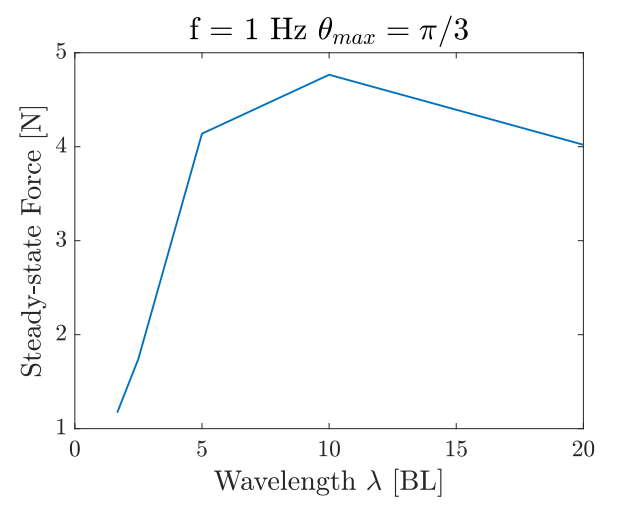

(b) Force in the swimming direction acting on the fish for different wavelengths at $1 \mathrm{~Hz}$

Fig. 18: Force in the swimming direction and velocity for different wavelengths at $1 \mathrm{~Hz}$

it adjusts wavelength and frequency to tune maneuvrability, power consumption and acceleration according to its needs. In Figure $18 \mathrm{a}$ it is evident that increasing the wavelength the steady-state velocity increases, then it reaches a maximum and, with an extremely high wavelength, it starts decreasing again. This happens because a longer wavelength means a greater wave propagation velocity, thus water is pushed backwards at a higher speed, giving more momentum to the fish. Nevertheless, a long wavelength also implies that the fin is flat and it pushes water more in a vertical direction than backward; therefore, it is more difficult to generate thrust. This result is consistent with the observation that batoid fishes with an oscillatory behavior, i.e. a longer wavelength, swim faster and their motion is suitable to roam across the oceans, whereas fishes with an undulatory behavior, i.e. a shorter wavelength, are featured by small velocities and live in coral reefs or in closed environments [17]. During steady-state swimming, force oscillates around a zero average value, as represented in Figure 8a. In Figure 18b the amplitude of these oscillations is represented for different wavelengths $\lambda$. Increasing the wavelength, the force is increased; however, as for the velocity, for very long wavelengths increasing further the wavelength does not have any positive effect on the generated thrust. When the fish swims with a large wavelength the behavior is very similar even though the wavelength are significantly different. This happens because the number of waves on the fin, the reciprocal of the wavelength is very similar. The amplitude of the oscillations of the power consumed at the steady-state by the fish and the efficiency of fish swimming are plotted in Figure 19 for different wavelengths $\lambda$. Swimming strategies involving a higher wavelength entail that the fin remains flatter, so the water is pushed more in a vertical direction. This is the reason why a shorter wavelength results in a more efficient swimming strategy. The efficiency is calculated averaging the power over a period, and it is meaningful only when the fish has reached the steady-state velocity.

\subsection{Effect of frequency}

For the study of the effect of frequency on swimming performaces it has been chosen to perform simulations with different frequencies and with wavelength of $2.5 \mathrm{BL} / \mathrm{s}$. This number has been chosen, since it is a good compromise between forward velocity at the steady-state and energy efficiency, as shown in the previous paragraph and because it is close to the real behavior of a cownose ray in nature [17,21]. In Figure 20 the forward velocity of the fish swimming with different 

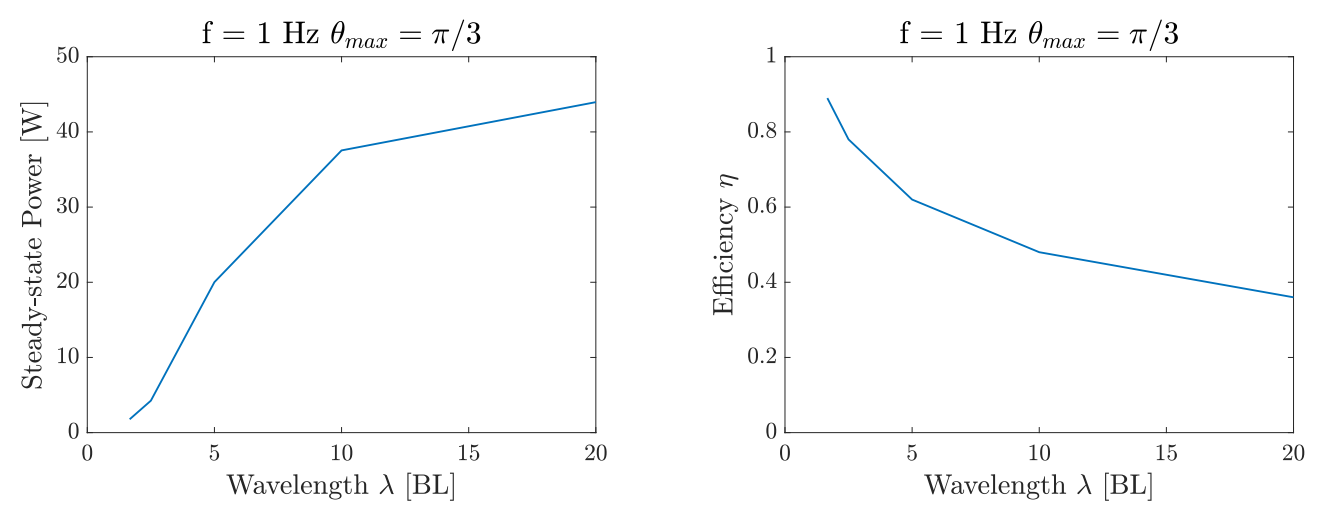

Fig. 19: Power and efficiency of the fish for different wavelengths at $1 \mathrm{~Hz}$

frequencies is presented and it can be observed that the steady-state velocity is proportional to frequency, consistently with the results obtained by Huang et al. [15]. The curves shown in Figure 20 have different lengths because the time unit of this graph is adimensionalized with respect to the period. Therefore, although they last approximately the same time, there is a different number of periods in each simulation. The force is proportional to the square of frequency, as it can be observed

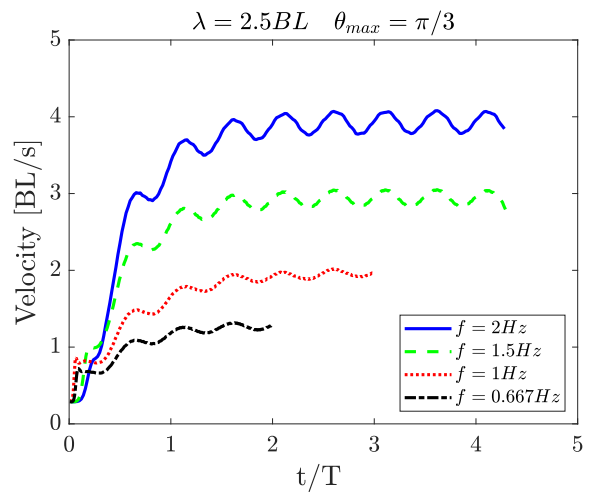

Fig. 20: Forward velocity of the swimming fish for different frequencies with $\lambda=2.5 \mathrm{BL}$

in Figure 21, where the dotted red line represents the fitting parabolic curve and the yellow area is the 95\% confidence interval of the fitting. The power is proportional to the cube of frequency, as shown in Figure 21, where the dotted red line represents the fitting parabolic curve and the yellow area is the $95 \%$ confidence interval of the fitting. This is expected since the velocity shows a linear dependency from frequency and force a quadratic dependence. This is a remarkable result because it means that the swimming of batoid fishes can be scaled with frequency in a linear fashion and, as a consequence, the efficiency is not affected by frequency variations, as shown in Figure 22. The effects of wavelength and frequency on swimming performances are summarized in Figure 23. It appears clear that the velocity increases with frequency and that it has a maximum for a particular wavelength, and that the efficiency only depends on wavelength. Therefore, in terms of energy consumption, the swimming of batoid fishes can reach high efficiencies, up to $89 \%$ for some swimming conditions and to achieve an acceleration it is more convenient to change the frequency instead of wavelength.

\subsection{Angle of attack}

Since the deformation of the fins of a cownose ray is featured by a small number of waves, about 0.4 [17], it is possible to consider every section of the fin as a heaving and pitching airfoil, and the angle of attack can be defined. The angle of attack $\alpha$ is the angle between the chord of the fin and the relative velocity of water with respect to the fin, as shown in Figure $24 \mathrm{a}$, where $\psi$ is the pitch angle of the fin section. The horizontal velocity of the fins $v_{x}$ is the same for every section of the fin, whereas the vertical velocity $v_{y}$ and the pitch angle $\psi$ are different for every section of the fin, since the fin tip motion has a larger amplitude than the fin root. Therefore, the angle of attack is different for every fin section. The angle of attack 

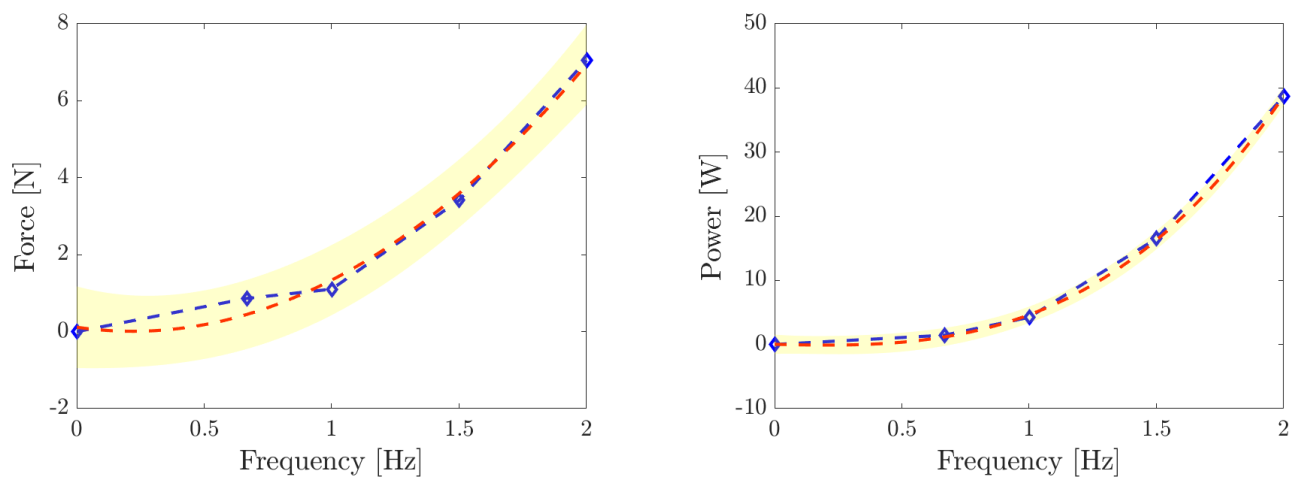

Fig. 21: Force and power of the fish for different frequencies with $\lambda=2.5 \mathrm{BL}$

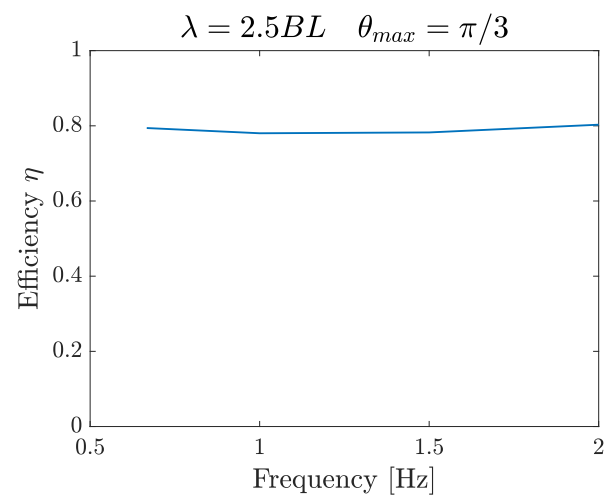

Fig. 22: Efficiency of the fish for different frequencies with $\lambda=2.5 \mathrm{BL}$
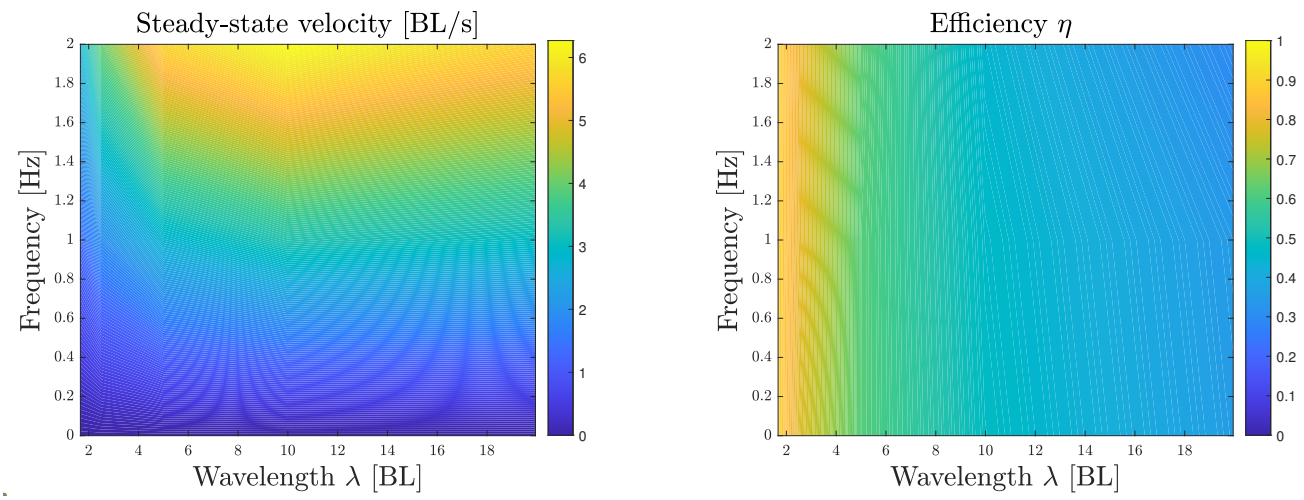

Fig. 23: Steady-state velocity and efficiency for different wavelengths and frequencies

is the difference between the angle of the relative velocity and the pitch angle $\psi$.

$$
\alpha=\psi-\arctan \frac{v_{y}}{v_{x}}
$$

Neglecting the motion of the fin section along the z-axis, i.e. out of the plane shown in Figure 24a, which is smaller than the other two components, the relative velocity $v_{r e l}$ has two components: a horizontal component $v_{x}$ equal to the forward velocity of the fish, and a vertical component $v_{y}$, which is $\dot{y}(s, t)$, the derivative of equation 4 . For a determined fin section the velocity $v_{y}$ is linearly dependent on frequency, and, as previously shown, also the forward velocity $v_{x}$ resulted to be 
proportional to frequency. On the other side, a variation of the wavelength does not affect the velocity $v_{y}$, but it changes significantly the forward velocity $v_{x}$, as shown in Figure 18a. Hence, the angle of the relative velocity does not depend on frequency, but only on wavelength of fin motion. Furthermore, the pitch angle $\psi$ too is independent of the frequency, and it is dependent only on wavelength. In particular, the larger is the wavelength the flatter is the fin and, as a consequence, the smaller is the pitch angle. Thus, the angle of attack of any section of the fin is a function of the wavelength of fin motion, and in Figure 24b the variation of the angle of attack of a section near the fin tip over a period is presented.

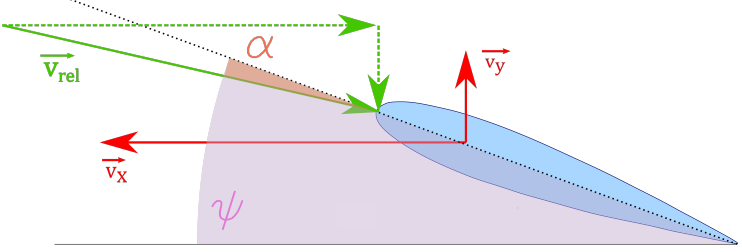

(a) Angle of attack of a fin section

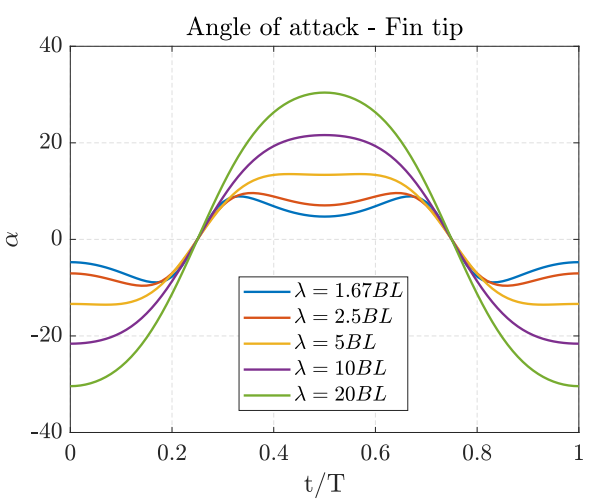

(b) Angle of attack of a fin section positioned at $90 \%$ of fin span over a period

Fig. 24: Angle of attack of a fin section positioned at $90 \%$ of fin span over a period

A change in wavelength has two conflicting effects on the angle of attack: the first is due to the variation of $\psi$, which would make $\alpha$ increase for smaller wavelengths, the second is due to the different forward velocity, which would make $\alpha$ decrease for shorter wavelengths. At the fin tip, the second effect is prevailing, so the angle of attack is larger for longer wavelengths. This means that when the fin is performing an upstroke the angle of attack is negative, whereas it is positive during a downstroke. The angle of attack is also a key parameter governing the dynamics of the leading-edge vortex, and it is possible to observe that a LEV is formed only when the fish swims with a long wavelength, i.e. with a high angle of attack, in accordance with [36], which states that a high angle of attack is fundamental for the formation of the LEV.

\subsection{Strouhal number}

The Strouhal number for a swimming fish is defined as follows:

$$
S t=\frac{f A}{U},
$$

where $f$ is the flapping frequency, $A$ is the peak-to-peak amplitude of the trailing edge of the fin and $U$ is the swimming velocity [26]. For a batoid fish, the trailing edge amplitude is variable along the span of the fin, thus it is better to measure the vertical distance between two vortex cores directly from the wake. It results that this distance is the peak-to-peak amplitude of the farthest span of the fin before the beginning of the fin tip vortex. The Strouhal number for different wavelengths and frequencies is presented in Figure 25.

Since the steady-state velocity is proportional to frequency, the Strouhal number does not depend on frequency, whereas it varies with wavelength. The Strouhal number of the cownose ray swimming resulted to be between 0.2 and 0.4 for most of the simulated swimming modes. This result fits with observations in [7,26,37] and it is a remarkable confirmation of the ability of the model to catch and reproduce the real behavior of the cownose ray. Furthermore, for the simulation with $f=1 \mathrm{~Hz}$ and $\lambda=2.5 \mathrm{BL}$, the most typical parameters of swimming for the cownose ray, the Strouhal number is perfectly in the middle of this optimal range. For a greater wavelength, the Strouhal number decreases below 0.2 and the propulsive efficiency decreases. Conversely, for a short wavelength, the Strouhal number results to be higher and outside the optimal range, although the energy efficiency is the highest among all the simulations and the trend of the efficiency with respect to wavelength suggests that the efficiency can increase indefinitely reducing the wavelength. However, this is not possible in practice because it would induce a great deformation on the fin surface that could be impossible to achieve for a real fish. The Strouhal number influences also the formation of the LEV, as stated by [13], who investigated the vortices formed by a stingray. They have found that a LEV can form even though the stingray swims with an undulatory motion. However, they 

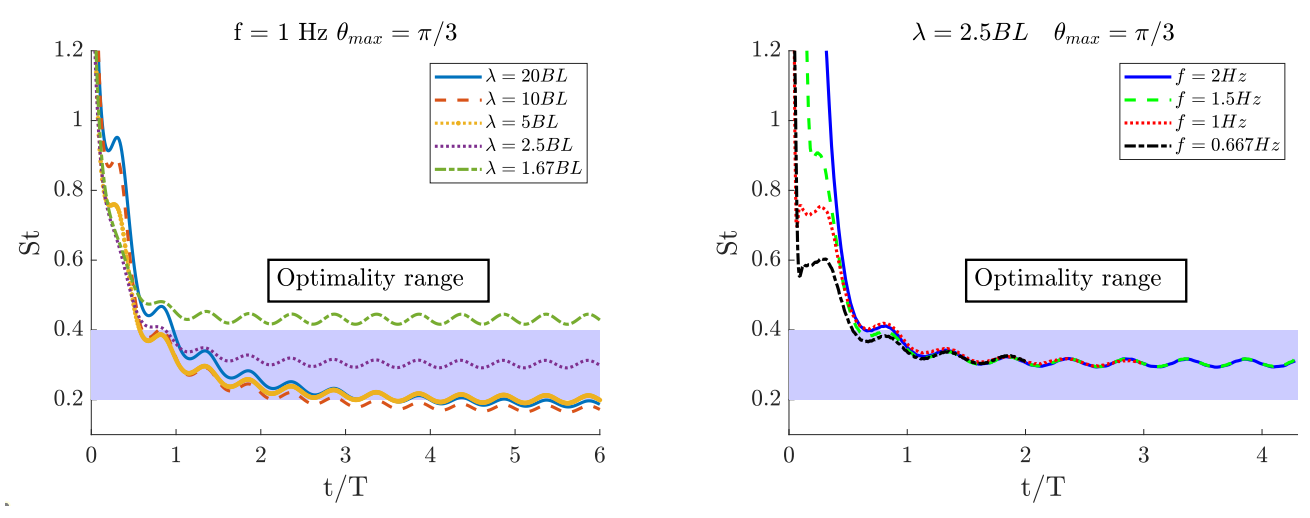

Fig. 25: Strouhal number for different wavelengths and frequencies

found that the formation of the leading-edge vortex is favored by a small Strouhal number ( 0.3$)$, which correspond to the same Strouhal numbers as for the cownose ray swimming with a large wavelength, resulting from this work. The stingray fin analyzed by [13] is featured by a significantly different geometry, and its motion is characterized by a smaller amplitude than the motion of the fins of a cownose ray.

\section{Conclusion}

A CFD model coupled with a dynamic solver has been developed, making use of a deformable overset grid. This method has been applied to simulate the swimming of a cownose ray with an imposed deformation on the fin, taken from the observations of the real fish made by biologists and it could be applied for each fish, once its motion is known.

The structure of the wake has been analyzed, highlighting the presence of vortices forming a reverse Karman street. This is related to thrust generation with an oscillatory motion and it is present in the wake of the majority of fishes. Furthermore, other vortices around the fin tips and near the center of the body have been put in evidence and they have been related to the fins motion. Such simulations have been carried out with different wavelengths and frequencies of fin motion and the swimming performances have been compared. The presence of a leading-edge vortex has been highlighted for fin motion with a long wavelength, nevertheless, such a long wavelength is not typical of the motion of a cownose ray, and its energy efficiency small. Despite the presence of a leading-edge vortex the main contribution to thrust is given by the travelling wave pushing water in the opposite direction.

The steady-state velocity is proportional to frequency and it is maximum for a particular value of wavelength. The energy efficiency for a self-propelled body has been defined in a novel way, it has been found that for the cownose ray it is remarkably high and that it does not depend on frequency, but only on wavelength. The calculated Strouhal number resulted to be in the same optimal range as other swimming and flying animals.

The developed model is a useful tool that will be used also in a future work to analyze the dynamics of maneuvering of batoid fishes and to understand the effect of spanwise wave propagation on the fin. The results obtained could be a starting point for the design of a bioinspired underwater robot and for the choice of its kinematics and its motion law, as they reveal the effect of different swimming strategies on motion performances.

\section{References}

[1] Dabiri, J., and Gordon, M., 2017. Animal Locomotion. Physical Principles and Adaptations. CRC Press. Taylor \& Francis Group, Boca Raton, FL, USA.

[2] Moored, K., Fish, F., Kemp, T., and Bart-Smith, H., 2011. "Batoid fishes: Inspiration for the next generation of underwater robots". Marine Technology Society Journal, 45(4), pp. 99-109.

[3] Salazar, R., Fuentes, V., and Abdelkefi, A., 2018. "Classification of biological and bioinspired aquatic systems: A review". Ocean Engineering, 148, pp. 75-114.

[4] Lighthill, M., 1969. "Hydromechanics of aquatic animal propulsion". Annual Review of Fluid Mechanics, 1, pp. 413446.

[5] Sparenberg, J., 2002. "Survey of the mathematical theory of fish locomotion". Journal of Engineering Mathematics, 44, pp. 395-448.

[6] Wu, T., 1960. "Swimming of a waving plate". Fluid Mechanics, 10, pp. 321-344.

[7] Fish, F., Schreiber, C., Moored, K., Liu, G., Dong, H., and Bart-Smith, H., 2016. "Hydrodynamic performance of aquatic flapping: Efficiency of underwater flight in the manta". Aerospace, 3. 
[8] Liu, G., Ren, Y., Zhu, J., and andH. Dong, H. B.-S., 2015. “Thrust producing mechanisms in ray-inspired underwater vehicle propulsion". Theoretical and Applied Mechanics Letters, 5, pp. 54-57.

[9] Zhan, J., Gong, Y., and Li, T., 2014. "Effect of angles of attack on the hydrodynamic forces of manta ray". In Proceedings of the Eleventh (2014) Pacific/Asia Offshore Mechanics Symposium, pp. 267-272.

[10] Sharp, N., Hagen-Gates, V., Hemingway, E., Syme, M., Via, J., Feaster, J., Bayandor, J., Jung, S., Battaglia, F., and Kurdila, A., 2014. "Computational analysis of undulatory batoid motion for underwater robotic propulsion". In Proceedings of the ASME 2014 4th Joint US-European Fluids Engineering Division Summer Meeting FEDSM2014, ASME, ed., pp. 1-8.

[11] Liu, X., Iwasaki, T., and Fish, F., 2013. "Dynamic modeling and gait analysis of batoid swimming". In 2013 American Control Conference, pp. 566-571.

[12] Wei-Shan, C., Zhi-Jun, W., Jun-Kao, L., Sheng-Jun, S., and Yang, Z., 2011. "Numerical simulation of batoid locomotion". Journal of Hydrodynamics, 23, pp. 594-600.

[13] Bottom, R. G., Borazjani, I., Blevins, E. L., and Lauder, G. V., 2016. "Hydrodynamics of swimming in stingrays: numerical simulations and the role of the leading-edge vortex". Journal of Fluid Mechanics, 788, pp. 407-443.

[14] Thekketil, N., Sharma, A., and Agrawal, A., 2020. "Three-dimensional biological hydrodynamics study on various types of batoid fishlike locomotion". Physiscal Review Fluids, 5(023101), pp. 1-26.

[15] Huang, H., Sheng, C., Wu, J., Wu, G., Zhou, C., and Wang, H., 2021. "Hydrodynamic analysis and motion simulation of fin and propeller driven manta ray robot". Applied Ocean Research, 108, p. 102528.

[16] Sfakiotakis, M., Lane, D. M., and Davies, J. B. C., 1999. "Review of fish swimming modes for aquatic locomotion". IEEE journal of Oceanic Engineering, 24(2), pp. 237-252.

[17] Rosemberger, L., 2001. "Pectoral fin locomotion in batoid fishes: Undulation versus oscillation". The Journal of Experimental Biology, 204, pp. 379-394.

[18] Cai, Y., Bi, S., Li, G., Hildre, H., and Zhang, H., 2018. "From natural complexity to biomimetic simplification. the realization of bionic fish inspired by the cownose ray". IEEE Robotics \& Automation Magazine.

[19] Cai, Y., Bi, S., and Zhang, L., 2010. "Design and implication of a bionic pectoral fin imitating cow-nosed ray". In The 2010 IEEE/RSJ International Conference on Intelligent Robots and Systems, pp. 3525-3529.

[20] Poulakis, G. R., 2013. "Reproductive biology of the cownose ray in the charlotte harbor estuarine system, florida". Marine and Coastal Fisheries: Dynamics, Management, and Ecosystem Science, 5, pp. 159-173.

[21] Russo, R., Blemker, S., Fish, F., and Bart-Smith, H., 2015. "Biomechanical model of batoid (skates and rays) pectoral fins predicts the influence of skeletal structure on fin kinematics: implications for bio-inspired design". Bioinspiration \& Biomimetics, 10.

[22] Moored, K., Dewey, P., Leftwich, M., Bart-Smith, H., and Smits, A., 2011. "Bioinspired propulsion mechanisms based on manta ray locomotion". Marine Technology Society Journal, pp. 110-118.

[23] Shi, G., Xiao, Q., Zhu, Q., and Liao, W., 2019. "Fluid-structure interaction modeling on a 3d ray-strengthened caudal fin". Bioinspiration \& Biomimetics, 14(3).

[24] Newmark, N., 1959. "A method of computation for structural dynamics". Journal of the Engineering Mechanics Division, 85(3), pp. 67-94.

[25] Gazzola, M., Argentina, M., and Mahadevan, L., 2014. "Scaling macroscopic aquatic locomotion". Nature Physics, 10.

[26] Eloy, C., 2012. "Optimal strouhal number for swimming animals". Journal of Fluids and Structures, 30, pp. 205-218.

[27] Siegel, S., Seidel, J., Cohen, K., and McLaughlin, T., 2007. "A cycloidal propeller using dynamic lift". In 37th AIAA Fluid Dynamics Conference and Exhibit, pp. 1-14.

[28] Anderson, J. M., Streitlien, K., Barrett, D. S., and Triantafyllou, M. S., 1998. "Oscillating foils of high propulsive efficiency". Journal of Fluid Mechanics, 360, pp. 41-72.

[29] Schnipper, T., Andersen, A., and Bohr, T., 2009. "Vortex wakes of a flapping foil". Journal of Fluid Mechanics, 633, pp. 411-423.

[30] Clark, R., and Smits, A., 2006. "Thrust production and wake structure of a batoid-inspired oscillating fin". Journal of Fluid Mechanics, 562, pp. 415-429.

[31] Dewey, P. A., Carriou, A., and Smits, A., 2012. "On the relationship between efficiency and wake structure of a batoid-inspired oscillating fin". Journal of Fluid Mechanics, 691, pp. 245-266.

[32] Borazjani, I., and Daghooghi, M., 2013. "The fish tail motion forms an attached leading edge vortex". Proceedings of the Royal Society B, $\mathbf{2 8 0}$.

[33] Lu, H., Yeoy, K. S., and Chew, C., 2018. "Effect of pectoral fin kinematics on manta ray propulsion". Modern Physics Letters B, 32, p. 1840025.

[34] Maertens, A., Triantafyllou, M., and Yue, D., 2015. "Efficiency of fish propulsion". Bioinspiration \& Biomimetics, 10.

[35] Bale, R., Hao, M., Bhalla, A., and Patankar, N., 2014. "Energy efficiency and allometry of movement of swimming and flying animals". Proceedings of the National Academy of Sciences, 111(21), pp. 7517-7521.

[36] Eldredge, J. D., and Jones, A. R., 2019. "Leading-edge vortices: Mechanics and modeling". Annual Review of Fluid 
Mechanics, 51, pp. 75-104.

[37] Taylor, G., 2018. "Simple scaling law predicts peak efficiency in oscillatory propulsion". PNAS, 115(32), pp. $8063-$ 8065.

\section{List of Figures}

1 CAD model of the cownose ray with its dimensions and the wing profiles that best approximate its shape . . 3

2 Representation of the coordinates $x$ and $s$, used in Eq. 1 , on the fin $\ldots \ldots \ldots \ldots \ldots \ldots \ldots$

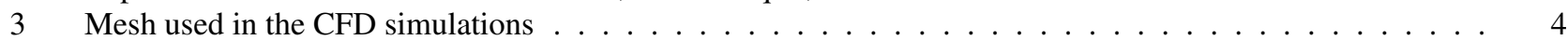

4 Domain of the CFD simulations, differentiating the two meshes with two colors . . . . . . . . . . 5

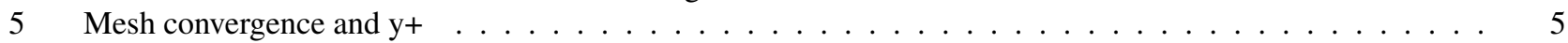

6 Force in swimming direction on the bottom side of the fish during upstroke . . . . . . . . . . . . . 6

7 Pressure distribution around the fin during upstroke and downstroke on a plane perpendicular to the fin span

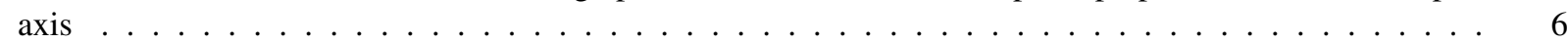

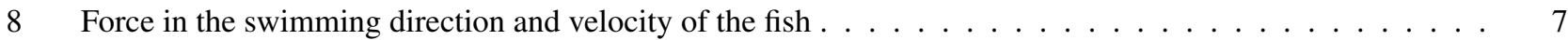

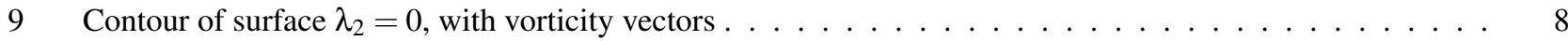

10 Sequence of time steps during a quarter of flapping cycle, highlighting the detachment of one of the vortices forming the reverse Karman street $\ldots \ldots \ldots \ldots \ldots \ldots$

11 Vortices in the wake . . . . . . . . . . . . . . . . . . . . . . 9

12 On the top velocity vectors; at the bottom velocity component in the swimming direction . . . . . . . . 9

13 Velocity vectors of the vortex around the fin tip during a downstroke . . . . . . . . . . . . . 10

14 Velocity vectors of the vortex on the central part of the fish body . . . . . . . . . . . . . . . . . 10

15 Velocity vectors and streamlines near the fin tip for $\lambda=1.67 B L \ldots \ldots \ldots \ldots \ldots \ldots$

16 Velocity vectors and streamlines near the fin tip for $\lambda=20 B L \ldots \ldots \ldots \ldots \ldots \ldots$

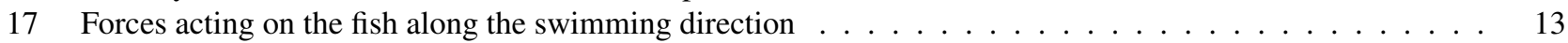

18 Force in the swimming direction and velocity for different wavelengths at $1 \mathrm{~Hz} \ldots \ldots \ldots$

19 Power and efficiency of the fish for different wavelengths at $1 \mathrm{~Hz} \ldots \ldots \ldots \ldots$. . . . . . . . . . 14

20 Forward velocity of the swimming fish for different frequencies with $\lambda=2.5 \mathrm{BL} \ldots \ldots \ldots \ldots$

21 Force and power of the fish for different frequencies with $\lambda=2.5 \mathrm{BL} \ldots \ldots \ldots \ldots \ldots$

22 Efficiency of the fish for different frequencies with $\lambda=2.5 \mathrm{BL} \ldots \ldots \ldots \ldots \ldots \ldots$

23 Steady-state velocity and efficiency for different wavelengths and frequencies . . . . . . . . . . . 15

24 Angle of attack of a fin section positioned at $90 \%$ of fin span over a period . . . . . . . . . . . . . . 16

25 Strouhal number for different wavelengths and frequencies . . . . . . . . . . . . . . . . . 17 